\title{
Comparison of three-dimensional digital technique with two-dimensional replica method for measuring marginal and internal fit of full coverage restorations
}

\author{
Mahya Hasanzade', Soudabeh Koulivand², Naeime Moslemian³, Marzieh Alikhasi** \\ 'Dental Research Center, Dentistry Research Institute, Department of Prosthodontics, School of Dentistry, Tehran University of \\ Medical Sciences, Tehran, Iran \\ ${ }^{2}$ Department of Prosthodontics, School of Dentistry, Shahid Beheshti University of Medical Sciences, Tehran, Iran \\ ${ }^{3}$ Department of Prosthodontics, School of Dentistry, Tehran University of Medical Sciences, Tehran, Iran \\ ${ }^{4}$ Dental Research Center, Dentistry Research Institute, Tehran University of Medical Sciences, Tehran, Iran
}

\begin{abstract}
PURPOSE. This study compared digital (reference point matching) and replica methods for measuring marginal and internal fit of full coverage restorations. MATERIALS AND METHODS. A maxillary left first molar typodent was fixed on to an aluminum base and prepared to receive all-ceramic full coverage restoration. The model was scanned with an intraoral scanner (CEREC Omnicam, Sirona, York, PA, USA). Twelve crowns were fabricated from lithium disilicate blocks (IPS emax CAD, Ivoclar Vivadent) and then crystalized. Marginal and internal fit of each restoration was measured by two examiners using replica and a new digital three-dimensional technique. Reliability between the two methods and two examiners was assessed by correlation and Cronbach's Alpha Coefficient $(P<.05)$. A Bland-Altman assessment for agreement was used to compare the two methods. RESULTS. Bland-Altman assessment showed that the mean of difference for marginal, absolute marginal, and axial gap was respectively $-1.04 \mu \mathrm{m},-41.9 \mu \mathrm{m}$, and $-29.53 \mu \mathrm{m}$ with limit of agreement (LOA) between -37.26 to $35.18 \mu \mathrm{m}$ for marginal, -105.85 to $22.05 \mu \mathrm{m}$ for absolute marginal and -80.52 to $22.02 \mu \mathrm{m}$ for axial gap. Positive correlation for repeatability $(P<.05)$ in determining marginal and internal gaps by the two examiners in both techniques was revealed. Reliability of both techniques in all sites of measurements was at least good $(0.8 \leq \alpha<0.9)$.
\end{abstract}

CONCLUSION. Both measuring techniques appeared highly reliable for evaluating fit of fixed dental restorations, while reference point matching provided higher values in axial and absolute marginal gap assessment. [J Adv

Prosthodont 2020;12:173-80]

KEYWORDS: Replica technique; Marginal adaptation; Internal adaptation; 3D measurement; Reliability

\section{INTRODUCTION}

All-ceramic fixed dental prostheses (FDP) are widely used due to their high aesthetic and biocompatibility. ${ }^{1}$ Besides

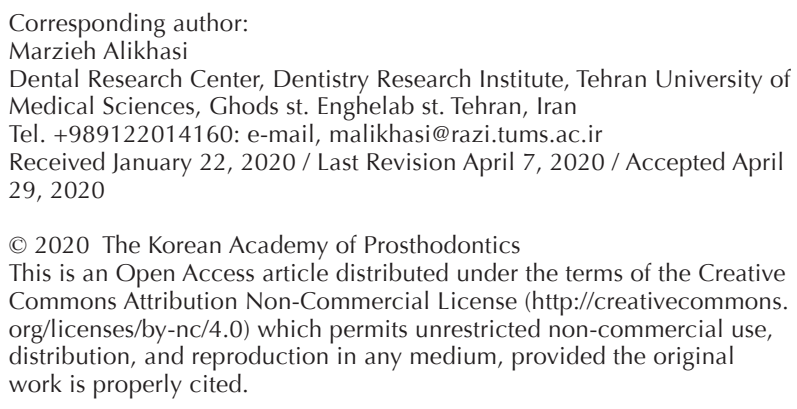

Dental Research Center, Dentistry Research Institute, Tehran University of Medical Sciences, Ghods st. Enghelab st. Tehran, Iran

Tel.+989122014160: e-mail, malikhasi@razi.tums.ac.ir

Received January 22, 2020 / Last Revision April 7, 2020 / Accepted April 29,2020

(C) 2020 The Korean Academy of Prosthodontics

This is an Open Access article distributed under the terms of the Creative Commons Attribution Non-Commercial License (http://creativecommons. org/licenses/by-nc/4.0) which permits unrestricted non-commercial use, distribution, and reproduction in any medium, provided the original work is properly cited.

aesthetic requirements, they should also have durability and biomechanical properties comparable to metal-ceramic restorations. $^{2}$ One of the major factors in long term success and clinical outcome of restored teeth is marginal adaptation. Marginal discrepancy can cause plaque retention and bacterial microleakage from the oral cavity that is detrimental to both tooth and supporting periodontal tissues. ${ }^{3,4} \mathrm{No}$ consensus exists on the exact maximum clinically acceptable marginal opening in the scientific literature. Christensen suggested 34 to $119 \mu \mathrm{m}$ as an acceptable range of marginal discrepancy. ${ }^{5}$ At present, most authors use the criteria reported by McLean of $120 \mu \mathrm{m}$, as the maximum acceptable marginal gap for long term success. ${ }^{6-8}$ Besides marginal discrepancy, the other factor that affects restoration seating, retention, and survival is internal discrepancy. Anadioti et al. 
showed that die spacer with $25-\mu \mathrm{m}$ thickness improved casting seating and increased retention. ${ }^{9}$ In addition, internal misfit can decrease the fracture resistance of all ceramic restorations. ${ }^{10}$

Different methods have been used in the previous studies to measure internal and marginal gap that could be categorized as 2-dimentinal measurements (2D), and 3-dimentional measurements (3D). The measurement method can be one of the reasons for the diversity of results among the studies in this field. Two-dimensional techniques measure the adaptation in limited points of direct view or cross-sectional views. Examples of 2D techniques include direct visualization with laser videography, ${ }^{11}$ profile projection, ${ }^{12}$ stereomicroscopy, ${ }^{13}$ and light microscopy. ${ }^{14}$ The most common 2D clinical method to investigate internal and marginal adaptation of a restoration is the replica technique. ${ }^{15,16}$ This method is non-invasive, simple, and affordable with acceptable accuracy. ${ }^{17}$ However, using silicone or resin to support the internal layer may cause dimensional changes in this layer and subsequent error in measurement of its thickness. ${ }^{18}$ Three dimensional techniques calculate the cement space between the intaglio surface of restoration and surface of teeth, resulting in numerous points available for measuring marginal and internal adaptation. Micro computed tomography $(\mathrm{CT})$, and triple scan protocol are among $3 \mathrm{D}$ measurements techniques. ${ }^{19}$ Although $3 \mathrm{D}$ digital methods were reported to be more accurate for measuring marginal and internal adaptation, ${ }^{19}$ they were only applied in laboratory studies. Triple-scan technique is a $3 \mathrm{D}$ method that consists of digitalization of the restoration, the abutment, and the assembly. By virtually superimposing scans of restoration and abutment based on the assembly scan, the misfit is measured, though the error of superimposing the surfaces is inevitable. ${ }^{19}$ Another approach to merge scans of two or more objects is reference point matching, in which the software uses some defined points to relate the scans. In this method, error of superimposition is limited and more accuracy is anticipated..$^{20,21}$ To the best of the authors knowledge, no study used this method to measure marginal or internal discrepancies of dental restorations. Before a new measurement technique is used in practice, the similarity of its measurement with values generated by the current measurement methods must be analyzed. Therefore, it is important to discover the relevance of the method used in the clinical studies and the one used for in vitro studies. The differences could be due to inherent variation in the measurement methods, the differences between the two techniques, and the variations between operators. A helpful parameter to investigate and quantify the agreement between two measurement methods is reliability.

The purpose of this in vitro study was to introduce and compare a new 3D method with the conventional replica technique in determining adaptation of $\mathrm{CAD} / \mathrm{CAM}$ single crowns. The secondary aim was to evaluate reliability of these two techniques. The null hypothesis was that there would be no difference between the two techniques in measuring the marginal and internal discrepancies.

\section{MATERIALS AND METHODS}

A maxillary left first molar typodent (Nissin Dental Prod. Inc., Tokyo, Japan) was attached on to a stellar shaped aluminum base with self-curing acrylic resin (Fastray, Harry J. Bosworth Co., Skokie, IL, USA). The tooth was prepared using a diamond rotary cutting instrument to receive all ceramic crowns. The preparation parameters were $2 \mathrm{~mm}$ occlusal reduction, $1 \mathrm{~mm}$ axial reduction and $360^{\circ}$ deep chamfer finish line. The model was scanned with an intraoral scanner (CEREC Omnicam, Sirona, York, PA, USA) Full contour restoration with minimum of $1 \mathrm{~mm}$ occlusal thickness, $0.8 \mathrm{~mm}$ axial thickness, and $60 \mu \mathrm{m}$ cement space was designed using CAD software (CEREC Premium SW 4.4, Dentsply Sirona, York, PA, USA). Twelve crowns were fabricated from lithium disilicate blocks (IPS emax CAD, Ivoclar Vivadent, Schaan, Liechtenstein) with a chairside 4-axis milling unit (Sirona Cerec MC XL, Sirona, York, PA, USA) and then crystalized in furnace (CEREC speed fire, Dentsply Sirona, York, PA, USA).

A clinician adjusted each restoration three times with light body silicone (GC Fit Checker, GC Corp, Tokyo, Japan) and a fine diamond bur (Rüthi, Switzerland) under magnification of a dental loop (HDL 2.5 Macro, Orascoptic, Madison, WI, USA). Marginal and internal adaptations of the twelve specimens were assessed with two techniques of replica technique and reference point matching by two examiners.

For measuring samples with replica technique, separating agent was applied over internal surfaces of the twelve restorations. Crowns were filled with Fit Checker (GC Corporation, Tokyo, Japan) and were seated on the prepared model. After setting of silicon layer, the crowns were removed while the Fit Checker layer was still attached to the teeth (Fig. 1A). A special tray was filled with heavy body additional silicone (Panasil heavy- Kettenbach GmbH \& Co. KG, Kansas, Germany) and placed over each prepared tooth with Fit Checker on it, and the stellar aluminum base. Tray was removed, and another heavy body additional silicone was injected over the Fit Checker layer to support it internally. The silicone material was carefully sectioned buccolingually and mesiodistally along the apexes of star base using a laser blade scalpel (Fig. 1B). Two calibrated clinicians measured the occlusal, midaxial, line angles, marginal and absolute marginal gap under a stereomicroscope (Leitz $\mathrm{GmbH}$, Oberkochen, Germany) at $\times 30$ magnification independently. Gap values were calculated according to Holmes et al..$^{22}$ by measuring the vertical distance from a point in the internal surface or the restoration margin to the axial wall or the margin of the prepared tooth.

Reference point matching (RPM) scan protocol was used as the second method for assessing marginal and internal discrepancies. Each model was scanned four times using a non-contact triple scanner (ATOS Core $5 \mathrm{Mp} 80 \mathrm{~mm}$; Rev.02; GOM, Braunschweig; Germany). First, each prepared tooth was scanned (Fig. 2A). After seating the restoration on the tooth, the second scan was made (Fig. 2B). Then an aluminum hex-shape index was attached to the occlusal surface of the crown and the third scan was made 

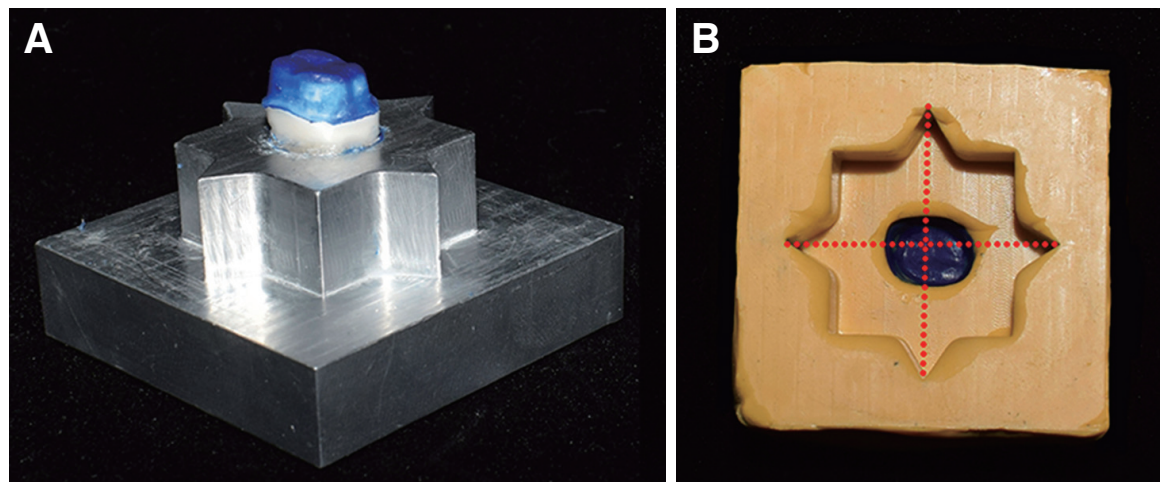

Fig. 1. (A) Fit Checker on prepared tooth, (B) Mesiodistal and buccolingual section across the edges of the star in the base.
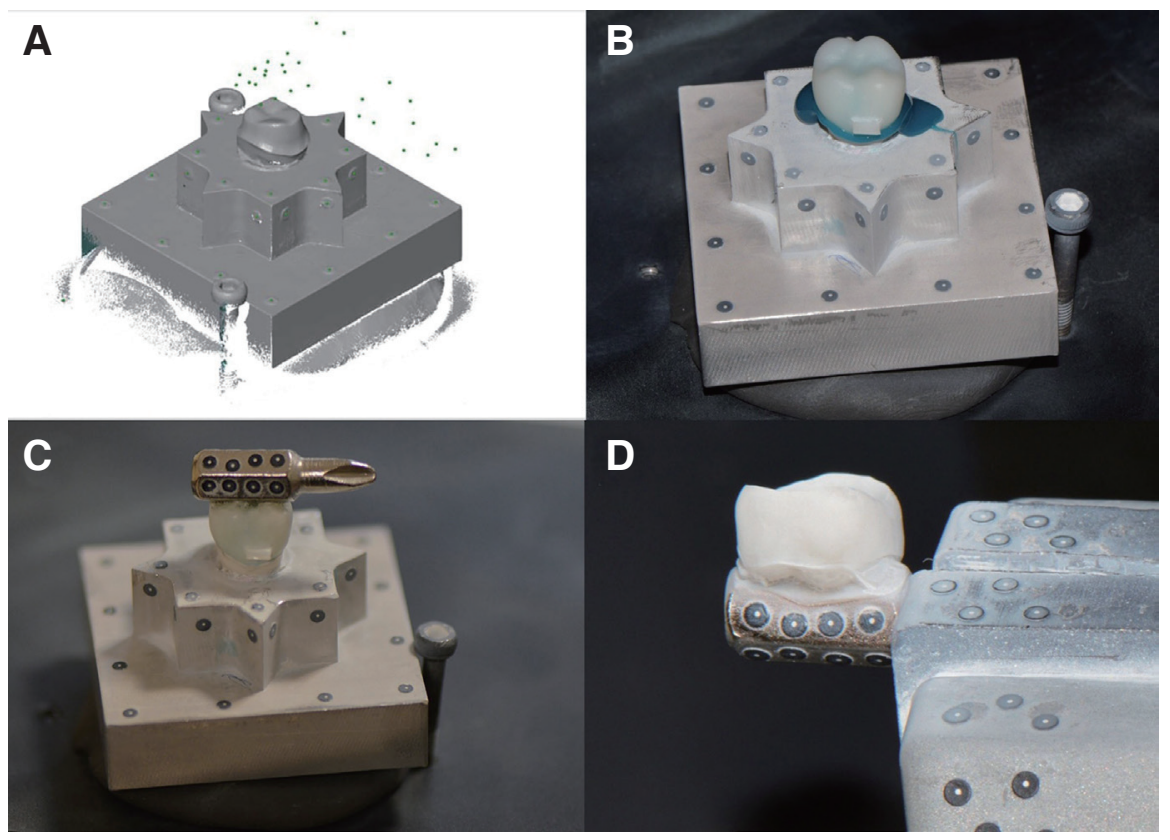

Fig. 2. (A) The tooth and its base are scanned, (B) The crown is placed on the prepared tooth and fixed with light body silicon material, (C) Hex shape index is attached on the occlusal surface, (D) The crown with the attached hex shape index is scanned.

from the stellar base and the restoration seated on the tooth (Fig. 2C). Finally, the restoration was removed from the tooth with the hex shape index in its place, and its internal and external surfaces with hex shape index were scanned (Fig 2D). The acquired data was processed with GOM software (GOM inspect v7.5, GOM mbH, Braunschweig, Germany) using the reference matching technique. Mesh data were transferred to a defined coordinate system by "3-2-1 alignment" that uses six 3D points to describe the coordinates. Mesiodistal and buccolingual intersecting edges along the apexes of stars in the aluminum bases served as 3D points and measurements were done in each of these two sections.
Same points as the first method were assessed by two examiners independently.

A Bland-Altman assessment for agreement was used to compare the two methods (with outlier removed). ${ }^{23} \mathrm{~A}$ range of agreement was defined as mean difference \pm 1.96 SD. It is expected that within the larger community and future measurements, the $95 \%$ of confidence interval between the two measurement methods will fall within this range.

Reliability between the two methods and two examiners was assessed by correlation and Cronbach's Alpha coefficient. All statistical analysis was performed using a statistical software (IBM SPSS Statistics v25; IBM Corp.). 


\section{RESULTS}

Mean, standard deviation, and mean differences of each examiner with the two techniques are shown in Table 1. Plots of the differences between the two methods using Bland-Altman test are shown in Fig. 3, Fig. 4, Fig. 5, and
Fig. 6. The smaller the difference between the two measurement methods (closer to zero) and the narrower the limits of agreement (LOA), the more similar the two methods are. For marginal gap, after omitting the outlier data in boxplot, mean of difference was $-1.04 \mu \mathrm{m}$ with LOA between -37.26 to $35.18 \mu \mathrm{m}$. One sample was omitted as outlier ${ }^{23}$ in assess-

Table 1. Descriptive statistics of each examiner, techniques and mean differences between the two examiners with two measurement techniques

\begin{tabular}{|c|c|c|c|c|c|c|c|c|c|c|c|}
\hline \multirow{3}{*}{\multicolumn{2}{|c|}{ Measurement technique }} & \multicolumn{10}{|c|}{ Discrepancy } \\
\hline & & \multicolumn{2}{|c|}{ Marginal } & \multicolumn{2}{|c|}{ Absolute marginal } & \multicolumn{2}{|c|}{ Axial } & \multicolumn{2}{|c|}{ Line angle } & \multicolumn{2}{|c|}{ Occlusal } \\
\hline & & Mean & SD & Mean & SD & Mean & SD & Mean & SD & Mean & SD \\
\hline \multirow{2}{*}{ Examiner 1} & Replica & 54.53 & 12.32 & $106.55^{\mathrm{a}}$ & 42.76 & $42.12^{d}$ & 8.85 & 102.18 & 28.61 & 250.36 & 46.84 \\
\hline & RPM & 56.79 & 29.48 & $162.54^{\mathrm{a}}$ & 51.08 & $71.37^{\mathrm{d}}$ & 19.42 & 129.43 & 39.69 & 248.50 & 64.74 \\
\hline \multirow{2}{*}{ Examiner 2} & Replica & 65.54 & 21.63 & $90.30^{b}$ & 27.83 & $46.10^{e}$ & 8.79 & 111.51 & 32.10 & 246.90 & 44.67 \\
\hline & RPM & 63.43 & 32.78 & $181.93^{b}$ & 56.50 & $73.66^{e}$ & 19.38 & 155.85 & 44.33 & 255.58 & 61.92 \\
\hline \multirow{2}{*}{ Total difference } & Replica & -11.00 & 11.91 & $16.24^{c}$ & 28.90 & -3.98 & 4.59 & $-9.33^{*}$ & 13.56 & 3.45 & 9.49 \\
\hline & RPM & -6.64 & 12.03 & $-19.39^{c}$ & 42.00 & -2.29 & 8.15 & $-26.41^{*}$ & 18.94 & -7.08 & 42.13 \\
\hline
\end{tabular}

RPM: reference point matching. ${ }^{*} P$ value $<.05$

Total difference $=$ values from examiner 1 - values from examiner 2 .

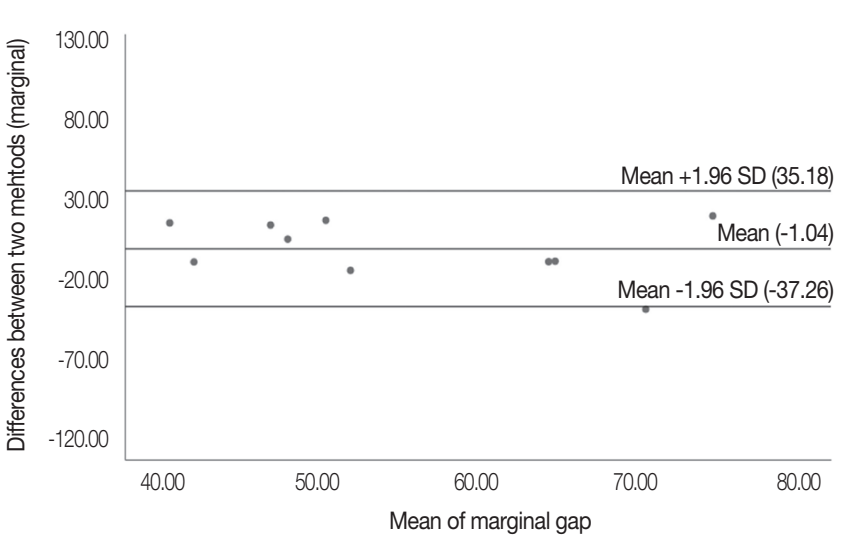

Fig. 3. Bland-Altman plot for reliability of marginal gap measurement.

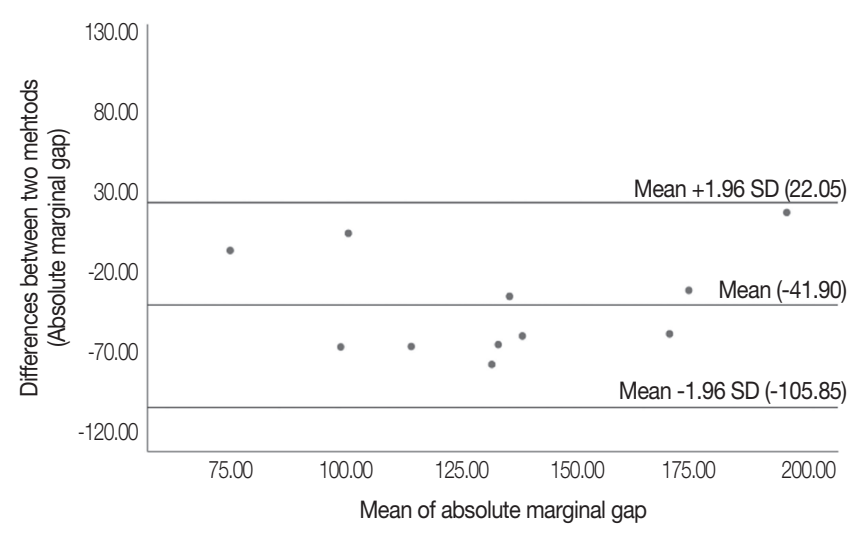

Fig. 4. Bland-Altman plot for reliability of absolute marginal gap measurement.

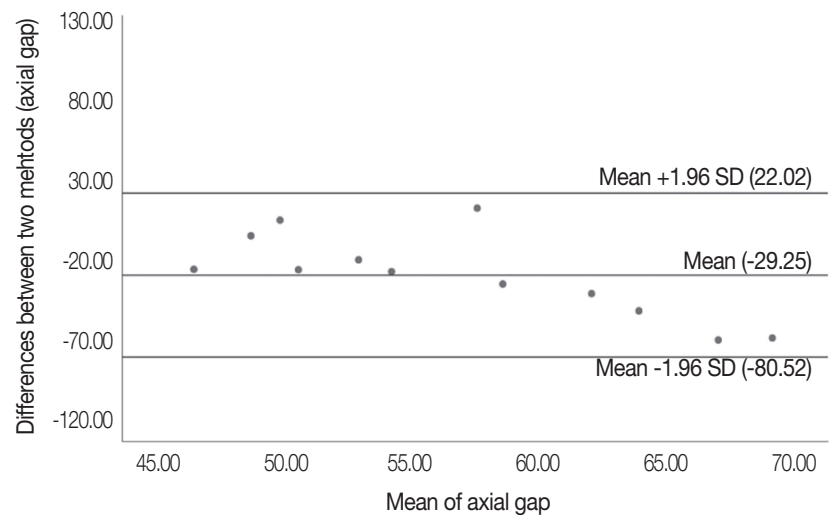

Fig. 5. Bland-Altman plot for reliability of axial gap measurement.

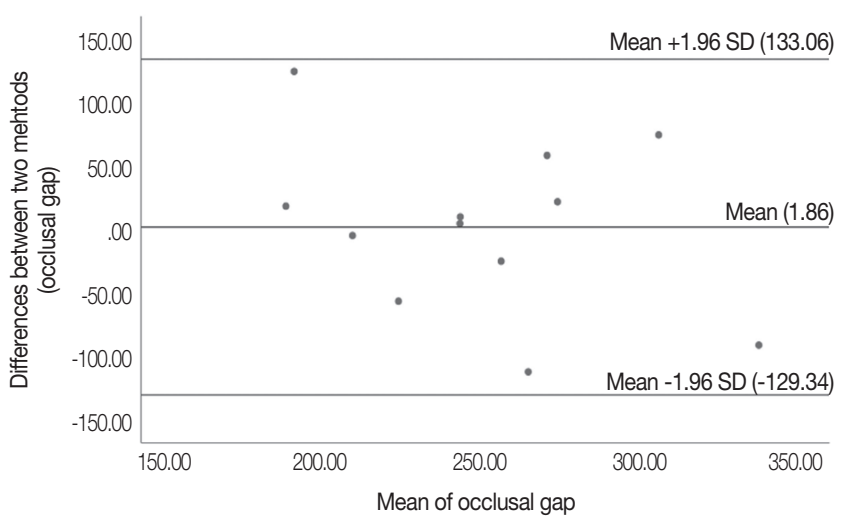

Fig. 6. Bland-Altman plot for reliability of occlusal gap measurement. 
Table 2. Correlation for the two examiners

\begin{tabular}{|c|c|c|c|c|c|}
\hline \multirow{2}{*}{ Measurement technique } & \multicolumn{5}{|c|}{ Discrepancy } \\
\hline & Marginal & Absolute marginal & Axial & Line angle & Occlusal \\
\hline \multirow{2}{*}{ Replica } & $0.896^{\star \star}$ & $0.743^{\star \star}$ & $0.864^{\star \star}$ & $0.907^{\star \star}$ & $0.980^{* \star}$ \\
\hline & $P<.001$ & $P=.006$ & $P<.001$ & $P<.001$ & $P<.001$ \\
\hline \multirow{2}{*}{ RPM } & $0.931^{\star \star}$ & $0.700^{\star}$ & $0.912^{\star \star}$ & $0.904^{\star \star}$ & $0.780^{\star \star}$ \\
\hline & $P<.001$ & $P=.011$ & $P<.001$ & $P<.001$ & $P=.003$ \\
\hline
\end{tabular}

RPM: reference point matching.

*. Correlation is significant at the 0.05 level (2-tailed).

${ }^{* *}$. Correlation is significant at the 0.01 level (2-tailed).

Table 3. Reliability of the two techniques

\begin{tabular}{|c|c|c|c|c|c|}
\hline \multirow{2}{*}{ Measurement technique } & \multicolumn{5}{|c|}{ Discrepancy } \\
\hline & Marginal & Absolute marginal & Axial & Line angle & Occlusal \\
\hline Replica & 0.871 & 0.809 & 0.927 & 0.948 & 0.989 \\
\hline RPM & 0.961 & 0.821 & 0.954 & 0.947 & 0.876 \\
\hline
\end{tabular}

ing agreement in absolute marginal gap, and then the result showed $-41.9 \mu \mathrm{m}$ mean difference and LOA between -105.85 to $22.05 \mu \mathrm{m}$. The mean difference between the two measurement techniques in axial site was $-29.53 \mu \mathrm{m}$ with LOA between -80.52 to $22.02 \mu \mathrm{m}$.

The results of Pearson Correlation test for examiners are shown in Table 2. Positive correlation for repeatability in determining marginal, absolute marginal, line angle, axial and occlusal discrepancy by two examiners in both techniques was revealed.

The statistical results of reliability by using Cronbach's alpha are shown in Table 3. Data in Table 3 shows excellent reliability $(0.9 \leq \alpha)$ of replica technique in measuring axial, line angle, and occlusal discrepancies and RPM technique in measuring marginal, axial, line angle and occlusal discrepancies. Reliability of the other site of measurements were $\operatorname{good}(0.8 \leq \alpha<0.9) .^{24}$

\section{DISCUSSION}

The purpose of this study was to assess the reliability and the level of agreement between the replica and the RPM methods used to measure the internal and marginal discrepancies of single unit lithium disilicate restorations. The null hypothesis of this study regarding producing similar marginal and internal discrepancy values with both techniques is partially rejected since there were some differences between two methods. However, the second null hypothesis was accepted, as there was no difference between reliability of replica and reference point matching technique.

Among all available 2D and 3D measurement methods, the most commonly used technique was direct-view technique followed by cross-sectioning method and impression replica technique. ${ }^{25}$ Replica technique measures the cement space by evaluating thickness of a light body additional silicon material that resemble the thickness of cement. The replica specimen is sectioned and internal fit can be evaluated under magnification of stereomicroscope. This method has some inherent deficiency, which limits its reliability and validity in measuring accurate level of discrepancies. Dimensional change of intermedium material, tearing of silicon material during sectioning, limited number of sections, lack of precision in determining the exact level of finishing line, and inability to specify restoration margin are among these deficiencies. $^{25}$ Additional disadvantages include difficulty in selecting the points where the marginal opening is to be measured. $^{25}$

Optical scanners offer an accurate and fast way for measuring small component in details. The use of this technology in measuring adaptation is based on the triple scan technique, which was presented in 2011. In this method, three scans are captured from prepared tooth, restoration seated on the tooth, and inner surface of the restoration. Then, data from the first and the third scans are related to each other with the second scan and finally the second scan is ommited. The key point in cement space measurement is how data from inner surface of restoration is correlated to data from outer surface of the tooth. Two methods exist for matching points: (1) reference point matching and (2) surface point matching. In surface point matching technique, the software merges the scans according to curvature and tangency of available surfaces and measures how close or 
similar one surface is to another, while reference point matching uses some coded and non-coded points. The coded points that match easily are utilized for determination of distance relation between points of the object and the noncoded points are utilized for 3D point reconstruction. ${ }^{20,21}$ The main limitation of triple scan method is using surface matching for merging data, so the accuracy of superimposition is dependent on the form of the crown and is different for each sample. In RPM method, the data from each scan are related to each other based on reference point matching, and this index is equal for all specimens. Therefore, the accuracy of alignment is equal for all specimens and is independent of crown form. In this method, a set of points that can be traced in space is often treated as reference to exploit global relationship in the point set. ${ }^{20,21}$

Use of RPM technique ensures exact 3D alignment of a restoration attached to the corresponding die. Although this technique does not include replica technique's boundaries such as limited number of cross-sections used for the measurement of discrepancy between the crown and the prepared tooth. Groten et al. ${ }^{26}$ indicated that the minimum number measurement points in non-clinical assessments should be at least 50. Digital workflow establishes three dimensional and unlimited virtual cross-sections for evaluating marginal and internal discrepancies.

Both techniques used in our study are noninvasive and do not necessitate destruction of restoration for measuring internal or marginal discrepancies. These characteristics are important factors for applying methods in clinical and in vitro investigations.

Our result of agreement showed that differences between the two methods is close to zero for marginal gap and the range of agreement is +35.18 to $-37.26 \mu \mathrm{m}$. Considering $120 \mu \mathrm{m}$ as the acceptable clinical gap for cement space, this range of agreement could be clinically acceptable. For absolute marginal gap, this level of agreement is between +22.05 to $-105.85 \mu \mathrm{m}$, which could result in overestimating or underestimating the real distance beyond the acceptable threshold. The mean difference of two methods for absolute marginal gap is $-41.9 \mu \mathrm{m}$ that is considerably far from zero. These results indicate that replica technique is underestimating the values for absolute marginal gap, which is probably due to the fact that level of finish line and the restoration is not clearly obvious in this method. However, both techniques were reliable in repeated measurements by two examiners evaluating all sites of measurement.

Boitelle et al. ${ }^{19}$ compared replica technique and triplescan protocol in assessing marginal discrepancy of zirconia copings. They concluded that both techniques are reliable, and triple-scan method provides significantly higher values of marginal fit; however, the coefficient of variation was lower for triple scan protocol than that of the replica method. In agreement to Boitelle et al.'s results, the present study indicated the reliability of both techniques. The results showed that the mean values of marginal fit varied between 54.53 and $65.54 \mu \mathrm{m}$ with the replica technique and between 56.79 and $63.43 \mu \mathrm{m}$ with the RPM technique. Differences in marginal gap assessment results can be due to different scan protocol between the two studies. Another study by Son et al. ${ }^{27}$ compared five marginal and internal fit evaluation approaches for fixed restoration, including triple scan and replica techniques. Marginal gap obtained from the replica technique revealed a significant difference with a low value. In this study, marginal gap was assessed without assessing absolute marginal gap and the scan protocol was different from our method.

In addition to vertical discrepancies widely disscussed in other stuties, Holmes et al. described horizontal discrepancy that categorized underextention and overextension of restorations. ${ }^{22}$ An underextended margin is the perpendicular distance from the marginal gap to the cavosurface angle of the tooth and an overextended margin is the perpendicular distance from the marginal gap to the casting margin. Determining exact level of finishing line and restoration margin are mandatory for calculating horizontal discrepancy, which is specified precisely in RPM technique. On the other hand, replica technique only recognizes vertical discrepancy.

Most authors still use the criteria of maximum $120 \mu \mathrm{m}$ discrepancy for long term success, reported by McLean in $1971 .{ }^{8}$ The results of this study revealed that the theory of acceptable marginal discrepancy within $120 \mu \mathrm{m}$ was not applied for all of the tested restorations, particularly for absolute marginal discrepancies values. De Almeida et al. ${ }^{28}$ and $\mathrm{Al}$ Hamad et al. ${ }^{29}$ also reported marginal gap of lithium disilicate crowns greater than $120 \mu \mathrm{m}$ and some other studies presented much lower values. ${ }^{30-33}$ Defining a standard value of marginal and internal gap for recently restorations made by advance technology is a necessity. Moreover, there is still no standard procedure to measure the adaptation of dental restorations. ${ }^{34}$ Several fitting evaluation techniques have been used by different investigations. ${ }^{27,35-37}$ The diversity of results of different studies can be due to study demography, type of research (in vitro or in vivo), sample size, material of restorations, and measurements protocol. ${ }^{14}$ As long as a there is a confounding factor of measurement method, assessment of the impact of other factors including the type of restoration and restoration material is questionable. One of the limitations of the current study is that repeatability of the methods was not measured and only the variations of different observers and techniques were calculated.

Moreover, extrapolating in vitro results to the in vivo studies should be made with caution. Despite of in vitro nature of reference point matching technique, good reliability with replica technique makes relating in vitro and in vivo studies possible. Further studies are required to inspect the correlation between RPM technique and other methods of fit assessment such as triple scan protocol.

\section{CONCLUSION}

Both replica and RPM measuring methods are highly reliable for evaluating adaptation of fixed dental restorations. It suggests that both techniques are applicable for measuring marginal and internal adaptation. However, the RPM meth- 
od provided values of absolute marginal discrepancies higher than those of replica technique, which seems to be closer to real value. Moreover, repeatability of both techniques with different examiners was acceptable.

\section{ORCID}

Mahya Hasanzade https://orcid.org/0000-0002-9114-2471

Soudabeh Koulivand bttps://orcid.org/0000-0001-9322-6495

Naeime Moslemian bttps://orcid.org/0000-0001-6868-0549

Marzieh Alikhasi https://orcid.org/0000-0002-2527-728X

\section{REFERENCES}

1. Beuer F, Naumann M, Gernet W, Sorensen JA. Precision of fit: zirconia three-unit fixed dental prostheses. Clin Oral Investig 2009;13:343-9.

2. Raigrodski AJ, Chiche GJ, Potiket N, Hochstedler JL, Mohamed SE, Billiot S, Mercante DE. The efficacy of posterior three-unit zirconium-oxide-based ceramic fixed partial dental prostheses: a prospective clinical pilot study. J Prosthet Dent 2006;96:237-44.

3. Att W, Komine F, Gerds T, Strub JR. Marginal adaptation of three different zirconium dioxide three-unit fixed dental prostheses. J Prosthet Dent 2009;101:239-47.

4. Kohorst P, Brinkmann H, Li J, Borchers L, Stiesch M. Marginal accuracy of four-unit zirconia fixed dental prostheses fabricated using different computer-aided design/computer-aided manufacturing systems. Eur J Oral Sci 2009;117:31925.

5. Christensen GJ. Marginal fit of gold inlay castings. J Prosthet Dent 1966;16:297-305.

6. Gonzalo E, Suárez MJ, Serrano B, Lozano JF. A comparison of the marginal vertical discrepancies of zirconium and metal ceramic posterior fixed dental prostheses before and after cementation. J Prosthet Dent 2009;102:378-84.

7. Euán R, Figueras-Álvarez O, Cabratosa-Termes J, Brufau-de Barberà M, Gomes-Azevedo S. Comparison of the marginal adaptation of zirconium dioxide crowns in preparations with two different finish lines. J Prosthodont 2012;21:291-5.

8. McLean JW, von Fraunhofer JA. The estimation of cement film thickness by an in vivo technique. Br Dent J 1971;131:10711.

9. Anadioti E, Aquilino SA, Gratton DG, Holloway JA, Denry IL, Thomas GW, Qian F. Internal fit of pressed and computer-aided design/computer-aided manufacturing ceramic crowns made from digital and conventional impressions. J Prosthet Dent 2015;113:304-9.

10. Tuntiprawon M, Wilson PR. The effect of cement thickness on the fracture strength of all-ceramic crowns. Aust Dent J 1995;40:17-21.

11. May KB, Russell MM, Razzoog ME, Lang BR. Precision of fit: the Procera AllCeram crown. J Prosthet Dent 1998;80:394404.

12. Quintas AF, Oliveira F, Bottino MA. Vertical marginal discrepancy of ceramic copings with different ceramic materials, finish lines, and luting agents: an in vitro evaluation. J
Prosthet Dent 2004;92:250-7.

13. Abdel-Azim T, Rogers K, Elathamna E, Zandinejad A, Metz M, Morton D. Comparison of the marginal fit of lithium disilicate crowns fabricated with CAD/CAM technology by using conventional impressions and two intraoral digital scanners. J Prosthet Dent 2015;114:554-9.

14. Nawafleh NA, Mack F, Evans J, Mackay J, Hatamleh MM. Accuracy and reliability of methods to measure marginal adaptation of crowns and FDPs: a literature review. J Prosthodont 2013;22:419-28.

15. Molin M, Karlsson S. The fit of gold inlays and three ceramic inlay systems. A clinical and in vitro study. Acta Odontol Scand 1993;51:201-6.

16. Falk A, Vult von Steyern P, Fransson H, Thorén MM. Reliability of the impression replica technique. Int J Prosthodont 2015;28:179-80.

17. Weaver JD, Johnson GH, Bales DJ. Marginal adaptation of castable ceramic crowns. J Prosthet Dent 1991;66:747-53.

18. Koulivand S, Ghodsi S, Siadat H, Alikhasi M. A clinical comparison of digital and conventional impression techniques regarding finish line locations and impression time. J Esthet Restor Dent 2020;32:236-43.

19. Boitelle P, Tapie L, Mawussi B, Fromentin O. Evaluation of the marginal fit of CAD-CAM zirconia copings: Comparison of 2D and 3D measurement methods. J Prosthet Dent 2018; 119:75-81.

20. Orteu JJ. 3-D computer vision in experimental mechanics. Opt Laser Eng 2009;47:282-91.

21. Belongie S, Malik J, Puzicha J. Shape context: A new descriptor for shape matching and object recognition. Adv Neur In. 2002, 24: p. 509-22.

22. Holmes JR, Bayne SC, Holland GA, Sulik WD. Considerations in measurement of marginal fit. J Prosthet Dent 1989;62:4058.

23. Kwak SK, Kim JH. Statistical data preparation: management of missing values and outliers. Korean J Anesthesiol 2017; 70:407-11.

24. Cortina JM. What is coefficient alpha? An examination of theory and applications. J Appl Psycho 1993;78:98-104.

25. Wolfart S, Wegner SM, Al-Halabi A, Kern M. Clinical evaluation of marginal fit of a new experimental all-ceramic system before and after cementation. Int J Prosthodont 2003;16:58792.

26. Groten M, Axmann D, Pröbster L, Weber H. Determination of the minimum number of marginal gap measurements required for practical in-vitro testing. J Prosthet Dent 2000;83: 40-9.

27. Son K, Lee S, Kang SH, Park J, Lee KB, Jeon M, Yun BJ. A comparison study of marginal and internal fit assessment methods for fixed dental prostheses. J Clin Med 2019;8. pii: E785.

28. de Almeida IG, Antunes DB, Braun NX, Restani A, Straioto FG, Galhano GA. CAD/CAM system influence marginal fit of different ceramic types? Indian J Dent Res 2019;30:127-9.

29. Al Hamad KQ, Al Quran FA, AlJalam SA, Baba NZ. Comparison of the accuracy of fit of metal, zirconia, and lithium disilicate crowns made from different manufacturing 
techniques. J Prosthodont 2019;28:497-503.

30. De Freitas BN, Tonin BSH, Macedo AP, Dos Santos TMP, De Mattos MDGC, Hotta TH, Matsumoto W. Adaptation accuracy of milled lithium disilicate crowns: A 2D and 3D microCT analysis. J Esthet Restor Dent 2020;32:403-9.

31. Sadid-Zadeh R, Li R, Miller LM, Simon M. Effect of fabrication technique on the marginal discrepancy and resistance of lithium disilicate crowns: An in vitro study. J Prosthodont 2019;28:1005-10.

32. Haddadi Y, Bahrami G, Isidor F. Accuracy of crowns based on digital intraoral scanning compared to conventional impression-a split-mouth randomised clinical study. Clin Oral Investig 2019;23:4043-50.

33. Elrashid AH, AlKahtani AH, Alqahtani SJ, Alajmi NB, Alsultan FH. Stereomicroscopic evaluation of marginal fit of E.max press and E.max computer-aided design and computer-assisted manufacturing lithium disilicate ceramic crowns: An in vitro study. J Int Soc Prev Community Dent 2019;9: 178-84.

34. Colpani JT, Borba M, Della Bona A. Evaluation of marginal and internal fit of ceramic crown copings. Dent Mater 2013; 29:174-80.

35. Oka Y, Sasaki J, Wakabayashi K, Nakano Y, Okamura SY, Nakamura T, Imazato S, Yatani H. Fabrication of a radiopaque fit-testing material to evaluate the three-dimensional accuracy of dental prostheses. Dent Mater 2016;32:921-8.

36. Schaefer O, Kuepper H, Thompson GA, Cachovan G, Hefti AF, Guentsch A. Effect of CNC-milling on the marginal and internal fit of dental ceramics: a pilot study. Dent Mater 2013; 29:851-8.

37. Park JM, Hämmerle CHF, Benic GI. Digital technique for in vivo assessment of internal and marginal fit of fixed dental prostheses. J Prosthet Dent 2017;118:452-4. 